\title{
Analysis of Photoassociation Spectra for Giant Helium Dimers.
}

\author{
J. Léonard A A. P. Mosk团 M. Walhout团 P. van der Straten $\$$ M. Leduc, and C. Cohen-Tannoudji \\ Ecole Normale Supérieure and Collège de France \\ Laboratoire Kastler Brossel, 24 rue Lhomond, 75231 Paris Cedex 05, France
}

(Dated: November 5, 2018)

\begin{abstract}
We perform a theoretical analysis to interpret the spectra of purely long-range helium dimers produced by photoassociation (PA) in an ultra-cold gas of metastable helium atoms. The experimental spectrum obtained with the PA laser tuned closed to the $2^{3} S_{1} \leftrightarrow 2^{3} P_{0}$ atomic line has been reported in a previous Letter. Here, we first focus on the corrections to be applied to the measured resonance frequencies in order to infer the molecular binding energies. We then present a calculation of the vibrational spectra for the purely long-range molecular states, using adiabatic potentials obtained from perturbation theory. With retardation effects taken into account, the agreement between experimental and theoretical determinations of the spectrum for the $0_{u}^{+}$purely long-range potential well is very good. The results yield a determination of the lifetime of the $2^{3} P$ atomic state.
\end{abstract}

PACS numbers: $34.20 . \mathrm{Cf}, 32.80 . \mathrm{Pj}, 34.50 . \mathrm{Gb}$

\section{INTRODUCTION}

Photoassociation (PA) spectroscopy is a powerful technique for acquiring information about the collisional properties of laser-cooled atoms. It has revealed a rich array of high-resolution spectroscopic data for alkali diatomic molecules [1] and provided a means of testing calculations of molecular dynamics. It has also led to good estimates of the s-wave scattering length [2, 3] that determines the behavior of ultra-cold dilute gases near quantum degeneracy.

The case of ${ }^{4} \mathrm{He}$ atoms in the metastable $2^{3} S_{1}$ state $\left(\mathrm{He}^{*}\right)$ is distinctive in that each atom carries a large internal energy of $20 \mathrm{eV}$. Photoassociation experiments with $\mathrm{He}^{*}$ were first demonstrated by Herschbach et al. with atoms trapped in a magneto-optical trap (MOT) [4]. However, the quantitative study of pair interactions has still to be completed. In particular, although Bose Einstein Condensation (BEC) has been achieved in $\mathrm{He}^{*}$ [5, 6], the scattering length remains uncertain. What is more, the accurate investigation of collisional properties [7] and of the dynamical behavior [8] of the ultra-cold $\mathrm{He}^{*}$ gas suffers from the uncertainty in the scattering length. In order to extract quantitative information from PA spectroscopy we have performed a new PA experiment starting from a magnetically trapped and evaporatively cooled metastable helium gas. We have thereby achieved greater state selectivity, higher density, and lower temperature than were obtained previously [4].

As a preliminary step toward the characterization of pair interactions, we have reported [9] the observation of purely long-range helium dimers produced by photoassociation of metastable helium atoms, with the PA laser

\footnotetext{
*Electronic address: leonard@lkb.ens.fr

†Permanent address: Dept. of science \& technology, and MESA+ institute, University of Twente, Netherlands.

${ }^{\ddagger}$ Permanent address: Calvin College, Grand Rapids, MI, USA.

$\S$ Permanent address: Utrecht University, Netherlands.
}

tuned close to the $2^{3} S_{1} \leftrightarrow 2^{3} P_{0}$ atomic line (see Figure 11). The novelty of these dimers is that they are produced from two highly excited atoms and therefore carry a huge internal energy of $40 \mathrm{eV}$. However, whereas one might expect the molecules to decay through autoionization, the primary decay mechanism is radiative. This fact allowed us to develop an original, "calorimetric" detection method based on the strong heating of the atomic cloud at resonant PA frequencies. Our preliminary model for the heating accounts for the conversion of a decaying molecule's vibrational kinetic energy into additional thermal energy within the cloud. Autoionization appears to have a negligible effect, probably because the inner turning points for these giant dimers are so far apart (around 150 bohr radii). Ionization is unlikely at such distances, so it is not surprising that these molecular states have not been observed with the ion detectors used in MOT experiments [4].

The present paper is meant to provide a theoretical complement to reference [9], which focused primarily on experimental methods and results. Because ${ }^{4} \mathrm{He}$ has no hyperfine structure, the theoretical approach is relatively simple as compared with alkali systems. Thus, giant helium dimers present an interesting case study, and we have attempted to emphasize important physical concepts in somewhat of a tutorial approach. In particular, a perturbative description of the electronic potentials is given, which provides a physical understanding of the formation of these molecules. Then, with a singlechannel adiabatic calculation of the effective molecular potentials we find purely long-range spectra that are in excellent agreement with those computed in [28] by more sophisticated techniques.

In Section II after a brief review of the experiment, we relate the molecular binding energy to the measured resonance frequency by subtracting shifts due to the magnetic trapping potential and the non-zero temperature of the atomic cloud. In particular, the free-bound character of the transitions leads to temperature-induced shifts which do not exist in the case of bound-bound transitions. Sec- 


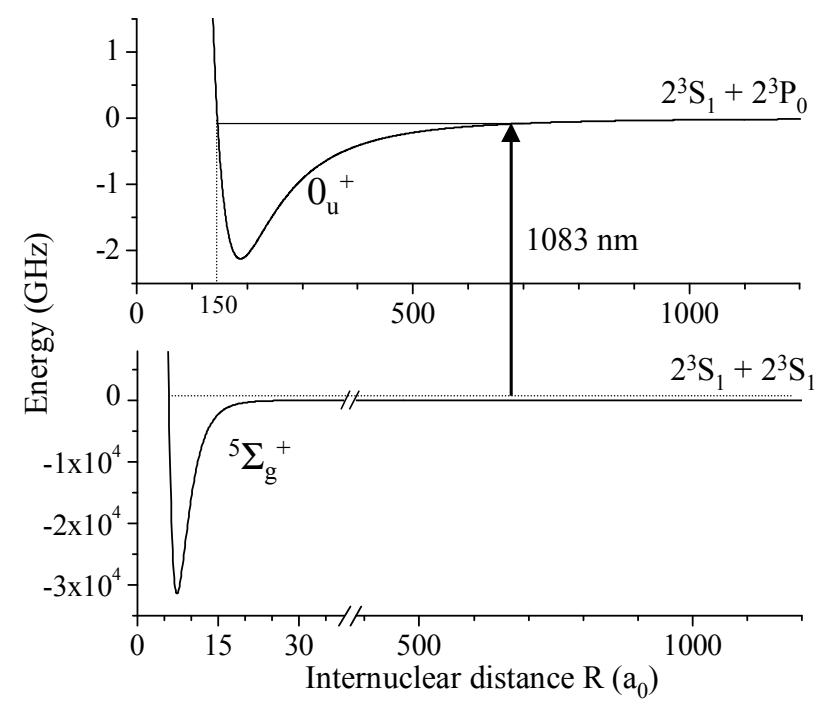

FIG. 1: a) Illustration of the principle of a photoassociation (PA) experiment. A free pair of metastable atoms is resonantly excited into a purely long-range $0_{u}^{+}$molecular bound state. The potential curve for the ${ }^{5} \Sigma_{g}^{+}$state is the one given by [10], the $0_{u}^{+}$is the one obtained by the calculation described in the text. Note the change in energy and length scales between the ${ }^{5} \Sigma_{g}^{+}$and the purely longrange $0_{u}^{+}$potential wells.

tion 111 describes the calculation of the long-range part of the $2^{3} S-2^{3} P$ molecular interaction potentials, as well as the theoretical values for the binding energies of the giant dimers. Our perturbative approach shows how purely long-range potential wells arise from the competition between the dipole-dipole interaction and the atomic fine structure. Finally, we compare both the experimental and theoretical determinations of the binding energies. With its high accuracy, the experiment provides a clear illustration of retardation effects in the electromagnetic interaction and of tiny corrections due to the vibrationinduced coupling between electronic and nuclear degrees of freedom. Moreover, it yields a measurement of the radiative decay rate $\Gamma$ of the atomic excited state $2^{3} P$ with an accuracy of $0.2 \%$.

\section{DERIVING THE BINDING ENERGIES FROM PA MEASUREMENTS}

\section{A. Acquisition of PA spectra}

We perform PA experiments with a cold metastable helium gas confined in a magnetic trap. The atomic cloud is cooled by RF-induced evaporation to a temperature in the $\mu \mathrm{K}$ range, just above the BEC transition [11]. The cloud is illuminated for a short period (0.1 to $10 \mathrm{~ms}$ ) by a low-intensity PA laser beam and then allowed to thermalize for a few hundred ms. It is subsequently released and then detected optically after a few-ms expansion time. Giant helium dimers are produced when a free

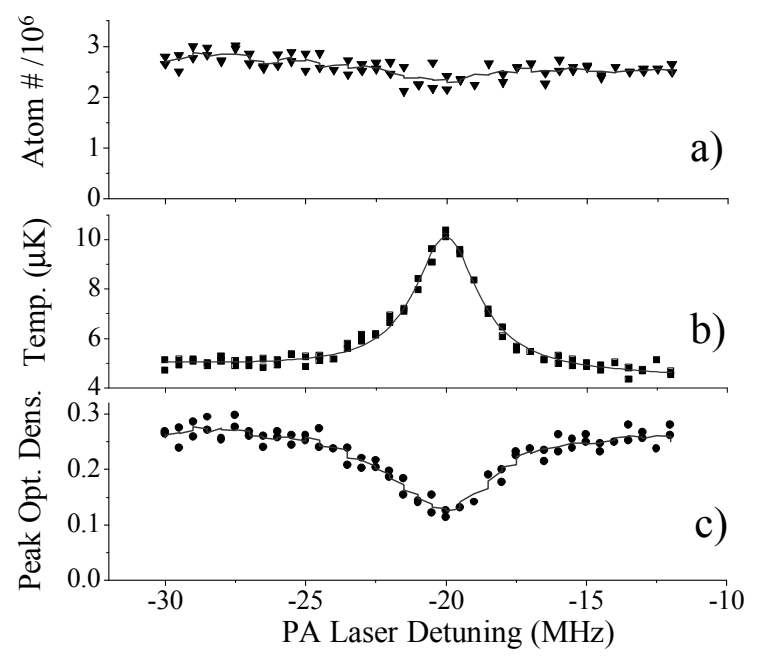

FIG. 2: Detection of the resonant formation of giant dimers in the $v=4$ vibrational state of the $0_{u}^{+}$potential well. After the PA laser pulse and further thermalization, the remaining atoms are detected optically: a) atom number, b) temperature in $\mu \mathrm{K}$ and c) peak optical density versus the PA laser detuning from the atomic $D_{0}$ line. Each point represents a new evaporated cloud after PA pulse illumination, thermalization and ballistic expansion. The curves in graphs a) and c) indicate the averaging of data over 5 adjacent points. The curve in graph b) is a Lorentzian fit to the data with a width of $2.8 \mathrm{MHz}$. Strong heating of the atomic cloud is observed when the PA laser is resonant with a molecular transition.

(unbound) pair of cold atoms absorbs a PA photon and is excited into a bound state of the purely long-range potential. This free-bound transition occurs when the PA laser is tuned red of the $2^{3} S_{1} \leftrightarrow 2^{3} P_{0}\left(D_{0}\right)$ atomic line (see Figure [1). Several resonance lines appear in the recorded temperature data, indicating that the formation of transient molecules results in the deposition of energy in the surrounding atomic cloud. Figure 2 illustrates the typical data obtained when we tune the PA laser in the vicinity of a bound state in the $0_{u}^{+}$potential well. Although few atoms are lost (Figure 2-a), a strong increase in temperature (Figure 2-b) and consequently a strong decrease in peak optical density (Figure 2-c) are monitored. Since the cloud is very cold (typically $5 \mu \mathrm{K}$ ), the excitation of relatively few molecules is enough to cause significant heating. Thus, the atomic cloud serves as a sensitive calorimeter capable of detecting the position of the molecular lines with an accuracy of $0.5 \mathrm{MHz}$. The quantitative study of the heating mechanism is in progress and will be published in a separate paper.

\section{B. Discussion of the various line shift mechanisms}

Acquiring experimental spectra consists in measuring the PA laser detunings at which molecular lines are resonantly excited in the magnetically trapped atomic cloud. For an accurate interpretation of the data, we need to take into account the correct lineshape function, which 
may include shifts and/or asymmetric broadening due to various mechanisms. We do so on the basis of the following calculation of the molecular binding energy, which emerges straightforwardly from the conservation of energy and momentum.

\section{Conservation of energy for a free-bound transition}

The energy $E_{i}$ of a pair of trapped atoms in the initial unbound state can be written:

$$
E_{i}\left(\overrightarrow{r_{1}}, \overrightarrow{r_{2}}, \vec{P}, \vec{p}_{r e l}\right)=\frac{\vec{P}^{2}}{4 m}+\frac{\vec{p}_{r e l}^{2}}{m}-\vec{\mu} \cdot\left(\vec{B}\left(\overrightarrow{r_{1}}\right)+\vec{B}\left(\overrightarrow{r_{2}}\right)\right),
$$

where $m$ is the mass of the He atom, $\vec{P}=\overrightarrow{p_{1}}+\overrightarrow{p_{2}}$ is the momentum of the pair's center of mass, $\vec{p}_{r e l}=\left(\overrightarrow{p_{1}}-\right.$ $\left.\overrightarrow{p_{2}}\right) / 2$ is the relative momentum, $\vec{B}\left(\overrightarrow{r_{1}}\right)$ and $\vec{B}\left(\overrightarrow{r_{2}}\right)$ are the magnetic field at the location of each atom, and $\vec{\mu}$ is the magnetic dipole moment of an atom in the $2^{3} S_{1}$ state (the Landé factor being 2 , we define $\mu=-2 \mu_{B}$, with the Bohr magneton $\mu_{B}<0$ ). In expression (11), we neglect any interaction energy between the two atoms. This will be justified below.

After the pair of atoms absorbs a photon with momentum $\hbar \vec{k}$ and frequency $\nu_{L}$, the binding energy $h b<0$ of the resulting molecule can be inferred from the conservation law for energy and momentum:

$$
\begin{aligned}
E_{i}\left(\overrightarrow{r_{1}}, \overrightarrow{r_{2}}, \vec{P}, \vec{p}_{r e l}\right)+h \nu_{L} & =\frac{\vec{P}_{M}^{2}}{4 m}+h\left(\nu_{0}+b\right) \\
\text { with } \vec{P}+\hbar \vec{k} & =\vec{P}_{M},
\end{aligned}
$$

where $\vec{P}_{M}$ is the final momentum of the molecule and $h \nu_{0}$ is the energy of the $D_{0}$ line (for an isolated atom in a zero magnetic field). The difference between the molecular binding energy and the PA laser detuning $\delta=\nu_{L}-\nu_{0}<0$ is thus given by:

$$
\begin{aligned}
h(b-\delta)= & -\hbar \vec{k} \cdot \frac{\vec{P}}{2 m}-\frac{\hbar^{2} k^{2}}{4 m} \\
& -\vec{\mu} \cdot\left(\vec{B}\left(\overrightarrow{r_{1}}\right)+\vec{B}\left(\overrightarrow{r_{2}}\right)\right)+\frac{\vec{p}_{r e l}^{2}}{m},
\end{aligned}
$$

Any dependence of the molecular level energy on the magnetic field (Zeeman effect) or on the density (mean field interaction of the molecule with the surrounding atomic and/or molecular cloud) is a priori included in $b$, which may therefore also depend on the position of the molecule.

Note that the relative kinetic energy term $\vec{p}_{r e l}^{2} / m$ in Equation (3) would not appear in the case of a boundbound transition, since it would be implicitly included in the initial binding energy. As it is always positive, it contributes an asymmetric lineshape, and consequently a mean shift 12. Also, the harmonic magnetic trapping potential contains quadratic terms which contribute to the inhomogeneous, asymmetric broadening and shift of the lines. However, the temperature is low enough that the asymmetric broadening terms remain much smaller than the natural lorentzian width. Thus, the only effect is a shift of the peak position of the lines, which can be calculated by averaging Equation (3) over the distribution function for the initial pair of free atoms.

\section{Initial distribution function of the free pair}

The distribution function for the pairs that undergo the PA transition is the thermal distribution for a pair of trapped atoms multiplied by the transition probability. According to the Franck-Condon overlap principle, the latter is proportional to the square of the overlap between the initial and final radial wave functions. Since the excited state is a bound state, the overlap is peaked at the Condon radius $R_{C}$ close to the classical outer turning point. According to Table III in Section IIII the transition occurs mainly for an internuclear distance $R_{C}=\left\|\overrightarrow{r_{1}}-\overrightarrow{r_{2}}\right\| \lesssim 50 \mathrm{~nm}$, which is much smaller than the size of the atomic cloud $(\sim 100 \mu \mathrm{m}$ at $T \sim 10 \mu \mathrm{K})$. This allows us to use the approximation $\overrightarrow{r_{1}} \simeq \overrightarrow{r_{2}} \simeq \vec{r}$ in Equations (11) and (3), where $\vec{r}$ is the center of mass of the pair. Furthermore, because the temperature is so low, the collision between two atoms occurs in the s-wave scattering regime, for which the relative angular momentum $\vec{p}_{r e l}=\hbar \vec{q}$ has no component orthogonal to the internuclear axis. Thus, the vectorial character of $\vec{p}_{r e l}$ can be ignored, since there is only one degree of freedom for the relative motion of the colliding atoms. For internuclear distances $R$ close to $R_{C}$, the radial part $u(R)$ of the ground state wave function can be approximated as $u(R) \propto \sin (q(R-a)) \propto q$ since $q R_{C} \ll 1$ (with $a$ representing the s-wave scattering length; see e.g. [1]). Finally, the distribution function for a pair of trapped atoms in the s-wave scattering regime absorbing a PA photon is found to be proportional to:

$$
\begin{gathered}
q^{2} \delta\left(\overrightarrow{r_{1}}-\overrightarrow{r_{2}}\right) \times \exp \left(-E_{i}\left(\overrightarrow{r_{1}}, \overrightarrow{r_{2}}, \vec{P}, q\right) / k_{B} T\right) . \\
\text { 3. Mean frequency shifts }
\end{gathered}
$$

i)Average over the center-of-mass momentum.

The first term in the right-hand side of Equation (3) is responsible for the Doppler profile. It produces no average shift, since there is a priori no correlation between the momenta of the two atoms and of the photon: $\langle\vec{k} . \vec{P}\rangle=0$. However, it is responsible for a symmetric broadening of the lines, which scales like $\sqrt{T}$ ( $T$, the temperature of the cold gas). In the microK range of temperature, this Doppler broadening turns out to be small compared with the natural lifetime broadening of the molecular states probed.

The second term in Equation (3) is the recoil energy of the molecule after absorbing the photon. In units of 
$h$, its numerical value is $\sim 21 \mathrm{kHz}$, which is well below our experimental accuracy. Therefore we neglect the corresponding shift.

ii) Average over the center of mass position.

Using expression (4), the average over the positions $\overrightarrow{r_{1}}$ and $\overrightarrow{r_{2}}$ turns out to be an average over the position $\vec{r}$ of the center of mass of the pair. The shift induced by the external trapping potential is thus calculated to be:

$$
\left\langle-\vec{\mu} \cdot\left(\vec{B}\left(\overrightarrow{r_{1}}\right)+\vec{B}\left(\overrightarrow{r_{2}}\right)\right)\right\rangle=2 \mu B_{0}+\frac{3}{2} k_{B} T,
$$

where $2 \mu B_{0}$ is twice the Zeeman shift of one atom at the center of the trap, and $3 k_{B} T / 2$ is the average of the harmonic trapping potential energy, according to the equipartition theorem for quadratic energy terms.

As already noted, the binding energy $h b$ a priori also depends on the center of mass position, and should therefore be averaged as well. However, we neglect this position dependence, since the effect of both the inhomogeneous magnetic field (molecular Zeeman effect) and density (atom-molecule interaction) turn out to be small compared with our experimental accuracy, as discussed below.

iii) Average over the relative momentum.

Making use of expression (4), we find the average of the relative kinetic energy term:

$$
\left\langle\frac{\hbar^{2} q^{2}}{m}\right\rangle=\frac{\int \frac{\hbar^{2} q^{2}}{m} q^{2} \exp \left(-\frac{\hbar^{2} q^{2}}{m k_{B} T}\right) d q}{\int q^{2} \exp \left(-\frac{\hbar^{2} q^{2}}{m k_{B} T}\right) d q}=\frac{3}{2} k_{B} T,
$$

where the denominator normalizes the distribution function. Let us mention that while there is only one degree of freedom for the relative momentum (in the s-wave scattering regime), our inclusion of the pair distribution function leads us coincidentally to the same $3 k_{B} T / 2$ that one finds when treating three classical degrees of freedom.

iv) Other shift mechanisms.

The mean-field interaction due to the surrounding medium on both the initial and final states of the transition can cause density-dependent shifts of the lines. As far as the initial pair of free atoms is concerned, the mean field interaction energy is $4 \pi \hbar^{2} \times n a / m$, where the atomic density $n<10^{14} \mathrm{~cm}^{-3}$, the s-wave scattering length $a<20 \mathrm{~nm} \mathrm{[5,} \mathrm{6],} \mathrm{and} m \sim 6.68 \times 10^{-27} \mathrm{~kg}$. In units of $h$, the upper bound for this mean-field interaction is less than $\sim 60 \mathrm{kHz}$, which is below our experimental accuracy and therefore negligible. The mean field energy shift of the final molecular state, which would appear as a density-dependent term in the experimental binding energy, has not been detected experimentally.

Finally, light-induced line shifts are completely negligible, since the spectra were measured with PA laser intensities well below the atomic saturation intensity.

v) Summary.

In our experiment, each molecular line produces a resonant increase in temperature as a function of PA detuning $\delta$. Each resonance line is fit by a Lorentzian. The fit's center frequency $\delta_{v}$ is taken to be the resonant frequency for excitation to vibrational level $v$. Accounting for the corrections described above, we infer the molecular binding energy $h b_{v}$ of this vibrational level to be:

$$
h b_{v} \simeq h \delta_{v}+2 \mu B_{0}+3 k_{B} T .
$$

\section{Experimental checks for the lineshifts}

We have measured $\delta_{v}, B_{0}$ and $T$ for the lines $v=0$ through $v=4$ in the $0_{u}^{+}$potential well, for $B_{0}=0.1$ to $\sim 10$ Gauss and for $T=1.5$ to $30 \mu \mathrm{K}$. The temperature of the gas was varied by changing the final RF frequency of the evaporation ramp above the critical temperature. Consequently, the atomic density was also varied from $n \sim 0.5 \times 10^{13}$ to $\sim 8 \times 10^{13}$ at $/ \mathrm{cm}^{3}$.

In Equation 7 the most important correction is due to $B_{0}$. Figure 3 shows the dependence on $B_{0}$ of the measured detuning $\delta_{v}$ of the $v=3$ line, after it is corrected for the temperature-induced effect $\left(3 k_{B} T\right)$. If the magnetic field is measured in units of $\mu B_{0}$, a linear fit to the data gives a slope of $-2.02 \pm 0.02$. Given Equation (7), the contribution of the initial pair of free cold atoms (the "ground" state), should be exactly $-2 \mu B_{0}$. A deviation from this value could be attributed to the contribution of the mean Zeeman effect of the molecular bound

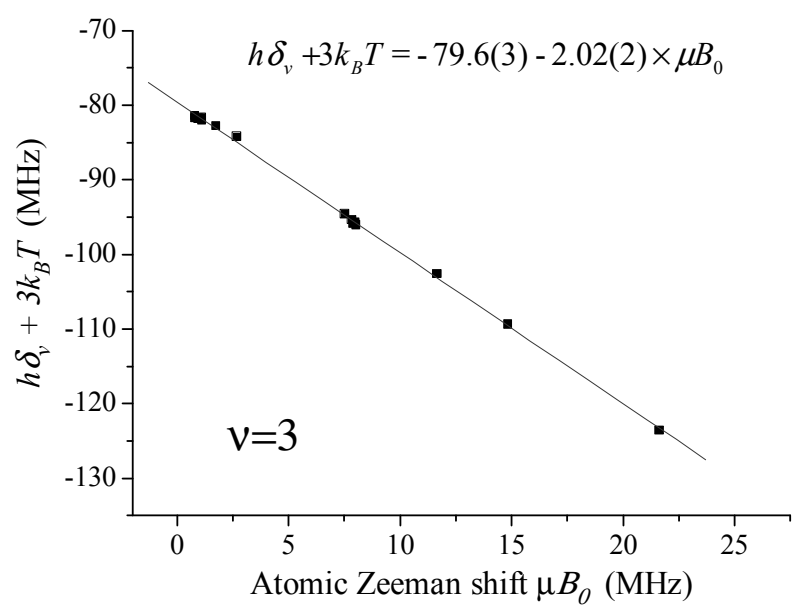

FIG. 3: Experimental determination of the binding energy in the $0_{u}^{+}$potential well for the vibrational level $v=3$ : illustration of the dependence of the measured detuning $\delta_{v}$ on the magnetic field $B_{0}$, after correction from the temperature-induced shift (see Equation (7). The slope of the linear fit is compatible with the expected dependence in $B_{0}$ (see in the text). 
("excited") state. As the $0_{u}^{+}$electronic state is non degenerate, the molecule cannot have any magnetic dipole moment except one induced by the molecular rotation, which is expected to be of the order of the nuclear magneton, or about three orders of magnitude smaller than $\mu_{B}$. Given the experimental accuracy and the range of magnetic field explored, the correspondingly small Zeeman effect would be difficult to measure. But our data permit us to set an upper bound of $0.02 \mu=0.04\left|\mu_{B}\right|$ on the molecular magnetic dipole moment. This result justifies neglecting the molecular Zeeman effect in the calculation of the mean line shifts.

Figure 4 displays the measured position of the $v=4$ line, corrected for the magnetically-induced shift $\left(2 \mu B_{0}\right)$, as function of the atomic cloud density. Data with (circles) and without (squares) the additional temperaturedependent correction are shown. The uncorrected data has been displayed in order to illustrate the importance of the temperature effect (up to $2 \mathrm{MHz}$ at $\sim 30 \mu \mathrm{K}$ ) as compared to the experimental accuray $(0.5 \mathrm{MHz})$. For this set of data, the density was increased simply by further evaporative cooling of the gas. Thus, higher density is associated with lower temperature, and the temperatureinduced shift indicated by the squares nearly vanishes for large density. It should be noted here that the size of the molecules (917 $a_{0} \sim 50 \mathrm{~nm}$, see Table III) is not vanishingly small compared with mean inter-atomic distance in the cloud $\left(\sim 260 \mathrm{~nm}\right.$ at $\left.6 \times 10^{13} \mathrm{at} / \mathrm{cm}^{3}\right)$. Under these conditions, one might expect to find a density- dependent shift due to the mean field interaction between the molecule and the surrounding atomic medium. However, no such shift is evident in our data after we apply the corrections for temperature and magnetic field. The er-

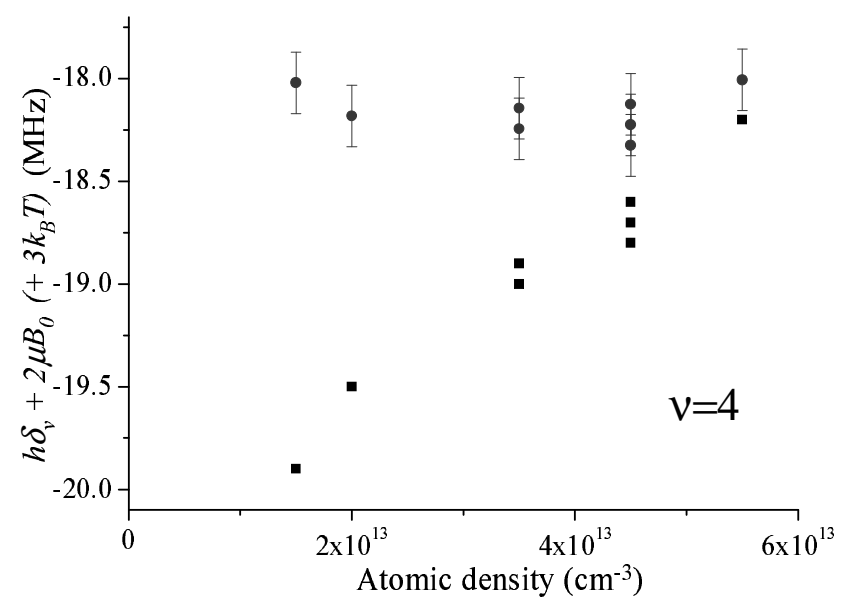

FIG. 4: Experimental determination of the binding energies in the $0_{u}^{+}$potential well: illustration, in the case of the vibrational level $v=4$, of the dependence of the measured detuning $\delta_{v}$ on the temperature and on the density, after correction from the magnetically-induced shift (see Equation (7)). Data are displayed before (squares) and after (circles) applying the temperaturedependent correction. Error bars include uncertainty in the measurements of $\delta, B_{0}$, and $T$. ror bars include experimental uncertainty in $\delta, B_{0}$ and $T$. Additional scatter of about $0.3 \mathrm{MHz}$ can be attributed to the uncertainty in the PA laser frequency lock. We have studied the stability of the experiment and the possible sources of systematic error in all achievable parameter ranges (accumulating many more data than are shown in Figure (4). We conclude that the binding energy for $v=4$ is $-18.2 \pm 0.5 \mathrm{MHz}$, in units of $h$.

Finally, from Figure 4 and from the $0.5 \mathrm{MHz}$ uncertainty, we can infer that the density-induced energy shift of the molecules must be smaller than $\sim 100 \mathrm{kHz}$ per $10^{13} \mathrm{~cm}^{-3}$ of density. Actually, the atomic Bose gas surrounding the molecule is near resonance and therefore has a permittivity that differs from the vacuum value. For an ideally homogeneous medium, the permittivity would enter in the resonant dipole potential [13], leading to a density-dependent term in the binding energy which would be at least a factor two above our upper bound. Since we do not detect this effect, we conclude our gas can not be considered as an homogeneous medium on the size scale of a molecule. This point may deliver important information about the three-particle correlation function in the atomic gas and would require further study, but it has not been investigated so far.

Similar data were registered for the other vibrational lines that we were able to measure. The experimental results for the binding energies are reported in Table \ Section [III]

\section{RO-VIBRATIONAL STRUCTURE OF THE GIANT DIMERS}

In order to interpret the measurements described above, we now develop the calculation of the long-range interaction of one atom in the $2^{3} S_{1}$ state, and another one in the $2^{3} P_{J=0,1,2}$ state. It happens that some of the resulting potential energy curves have minima at very large internuclear distance and support purely long-range bound states. In particular, the calculated spectrum of five vibrational states in the $0_{u}^{+}$potential will be shown to be in excellent agreement with our measurements.

\section{A. Electronic potential curves for the $2^{3} S+2^{3} P$ system with fixed nuclei \\ 1. Hamiltonian}

The general task for calculating molecular potentials in ${ }^{4} \mathrm{He}$ consists in solving the following Schrödinger equation [14]:

$$
\begin{aligned}
& \hat{H}\left|\psi_{\alpha}\right\rangle=\left(\hat{T}_{n}+\hat{T}_{e}+\hat{V}+\hat{H}_{r e l}\right)\left|\psi_{\alpha}\right\rangle=E_{\alpha}\left|\psi_{\alpha}\right\rangle(8) \\
& \text { where } \hat{T}_{n}=\sum_{k=1}^{2} \frac{\hat{\mathbf{p}}_{\mathbf{k}}^{2}}{2 M}, \hat{T}_{e}=\sum_{i=1}^{4} \frac{\hat{\mathbf{p}}_{\mathbf{i}}^{2}}{2 m} \\
& \text { and } \hat{V}=\hat{V}\left(\hat{\mathbf{r}}_{k}, \hat{\mathbf{r}}_{i}\right), \hat{H}_{r e l}=\hat{H}_{r e l}\left(\hat{\mathbf{r}}_{i}, \hat{\mathbf{s}}_{i}\right) .
\end{aligned}
$$


Here, $\left|\psi_{\alpha}\right\rangle$ is a stationary solution corresponding to a set of quantum numbers $\{\alpha\}$ to be detailed later. The hamiltonian written above appears as the sum of four terms $\hat{T}_{n}, \hat{T}_{e}, \hat{V}$ and $\hat{H}_{r e l}$ which represent respectively the kinetic energy of the two nuclei, the kinetic energy of the four electrons, the non relativistic interaction between the six charged particles, and the relativistic part of the hamiltonian. This operator is written as function of the positions of the nuclei $\hat{\mathbf{r}}_{k}$, and of the electrons $\hat{\mathbf{r}}_{i}$, and as function of the spin coordinates $\hat{\mathbf{s}}_{i}$ of the four electrons. The ${ }^{4} \mathrm{He}$ nuclei have no spin. To solve this very complicated problem, we adopt a perturbative approach, in which we consider the internuclear distance large enough that the interaction potential $\hat{V}$ can be treated as a perturbation of the system of two independent atoms $A$ and $B$. Thus the hamiltonian (8) is approximated as follows:

$\hat{H}=\hat{T}_{n}+\hat{H}_{0}(A)+\hat{H}_{0}(B)+\hat{H}_{f s}(A)+\hat{H}_{f s}(B)+\hat{U}(R)(9)$

where $\hat{H}_{0}$ and $\hat{H}_{f s}$ are respectively the non-relativistic and relativistic part of the hamiltonian for one isolated atom, and $\hat{U}(R)$ stands for the long-range electrostatic interaction between the two atoms, whose leading term is the retarded dipole-dipole interaction.

To describe long-range molecular interactions, we expand the molecular state in linear combinations of (entangled) atomic states (LCAO approximation). Moreover, according to the usual Born-Oppenheimer approximation we first consider only the electronic degrees of freedom while keeping the nuclei (more precisely, the atomic centers of mass) fixed. We then treat both the dipole-dipole interaction and the atomic fine structure as perturbations of the non-relativistic hamiltonian for two independent atoms. We write the two interactions in the basis set of states formed by the tensorial product of isolated non relativistic atomic states: $\{\mid$ atom $A$ : $\left.L_{A}, M_{L A} ; S_{A}, M_{S A}\right\rangle \otimes \mid$ atom $\left.\left.B: L_{B}, M_{L B} ; S_{B}, M_{S B}\right\rangle\right\}$. Considering one atomic orbital in the $2^{3} S$ state and another one in the $2^{3} P$ state, the space of states is of dimension 54. As the two nuclei are identical, the hamiltonian is unchanged under the inversion $\hat{I}_{e}$ of all the electrons with respect to the center of mass [15]. The operator $\hat{I}_{e}$ commutes with the hamiltonian (9) and has two eigenvalues $\omega= \pm 1$ with eigenstates labeled gerade $(g)$ and ungerade $(u)$ respectively.

\section{Retarded dipole-dipole interaction}

The dipole-dipole interaction $\hat{U}(R)$, first, only couples the orbital angular momenta of the two independent nonrelativistic atoms. It is diagonal in the Hund's case (a) basis set labelled $\left|{ }^{2 S+1} \Lambda_{u / g}\right\rangle$ (see e.g. 15, 16]). These states can be written as follows in the atomic basis:

$$
\begin{array}{r}
\left|{ }^{2 S+1} \Lambda_{u / g}\right\rangle=\frac{1}{\sqrt{2}}\left(1+\omega \hat{I}_{e}\right)\left|A: 0,0 ; B: 1, M_{L}\right\rangle \otimes\left|S, M_{S}\right\rangle \\
=\frac{1}{\sqrt{2}}\left(\left|A: 0,0 ; B: 1, M_{L}\right\rangle-\omega(-1)^{S}\left|A: 1, M_{L} ; B: 0,0\right\rangle\right) \\
\otimes\left|S, M_{S}\right\rangle .
\end{array}
$$

Here, $S$ is the total electronic spin of the molecule $(S=0$, 1 or 2$), \Lambda$ is the projection onto the molecular axis of the electronic orbital angular momentum of the molecule. In the Hund's case (a) basis, the retarded dipole-dipole interaction is respectively given by [16, 17]:

$$
-2 \omega(-1)^{S} C_{3} / R^{3} \times(\cos (k R)+k R \sin (k R)),
$$

$\omega(-1)^{S} C_{3} / R^{3} \times\left(\cos (k R)+k R \sin (k R)-(k R)^{2} \cos (k R)\right)$,

for ${ }^{2 S+1} \Sigma_{u / g}$ states (10a), and ${ }^{2 S+1} \Pi_{u / g}$ states (10b). The coefficient $C_{3}$ is related to the atomic dipole matrix element $d=<2^{3} P\left|\hat{d}_{z}\right| 2^{3} S>$, and thus to the radiative life time $1 / \Gamma$ of the atomic transition:

$$
C_{3}=\frac{|d|^{2}}{4 \pi \varepsilon_{0}}=\frac{3}{4} \hbar \Gamma\left(\frac{\lambda}{2 \pi}\right)^{3},
$$

with $\varepsilon_{0}$ the vacuum permittivity. The fine structure splitting is small enough that we assume the three atomic lines of interest $\left(2^{3} S_{1} \leftrightarrow 2^{3} P_{J=0,1,2}\right)$ have the same wavelength $\lambda=1083.3 \mathrm{~nm}$ within $0.1 \mathrm{~nm}$. The radiative decay rate $\Gamma=2 \pi \times 1.6248 \mathrm{MHz}$ can be inferred from $\lambda^{2}$ and from an accurate calculation of the oscillator strength of the atomic transition [18]. Finally, $C_{3}$ is found to be $C_{3}=6.405$ atomic units, within a relative uncertainty of $5 \times 10^{-4}$.

\section{Fine structure coupling}

We next consider the relativistic part of the hamiltonian, $\hat{H}_{f s}(A)+\hat{H}_{f s}(B)$, which is diagonal in the Hund's case (c) basis (by definition of Hund's case (c), see e.g. [15]), with three eigenvalues corresponding to the three states $2^{3} S_{1}+2^{3} P_{J=0,1,2}$. The eigenstates can only be characterized by the projection $\Omega$ of the total electronic angular momentum (orbital and spin) on the molecular axis 15 . In ${ }^{4} \mathrm{He}$ the atomic fine structure can be modeled using the following operator:

$$
\hat{H}_{f s}=\alpha \vec{L} . \vec{S}+\beta(\vec{L} . \vec{S})^{2},
$$

where $\vec{L}$ and $\vec{S}$ are the atomic orbital and spin angular momenta. In addition to the usual spin-orbit coupling, spin-spin magnetic dipole interaction between the two electrons is an important effect in helium [19], leading to a non equidistant splitting of the fine structure levels. In our model, the constants $\alpha$ and $\beta$ are determined 


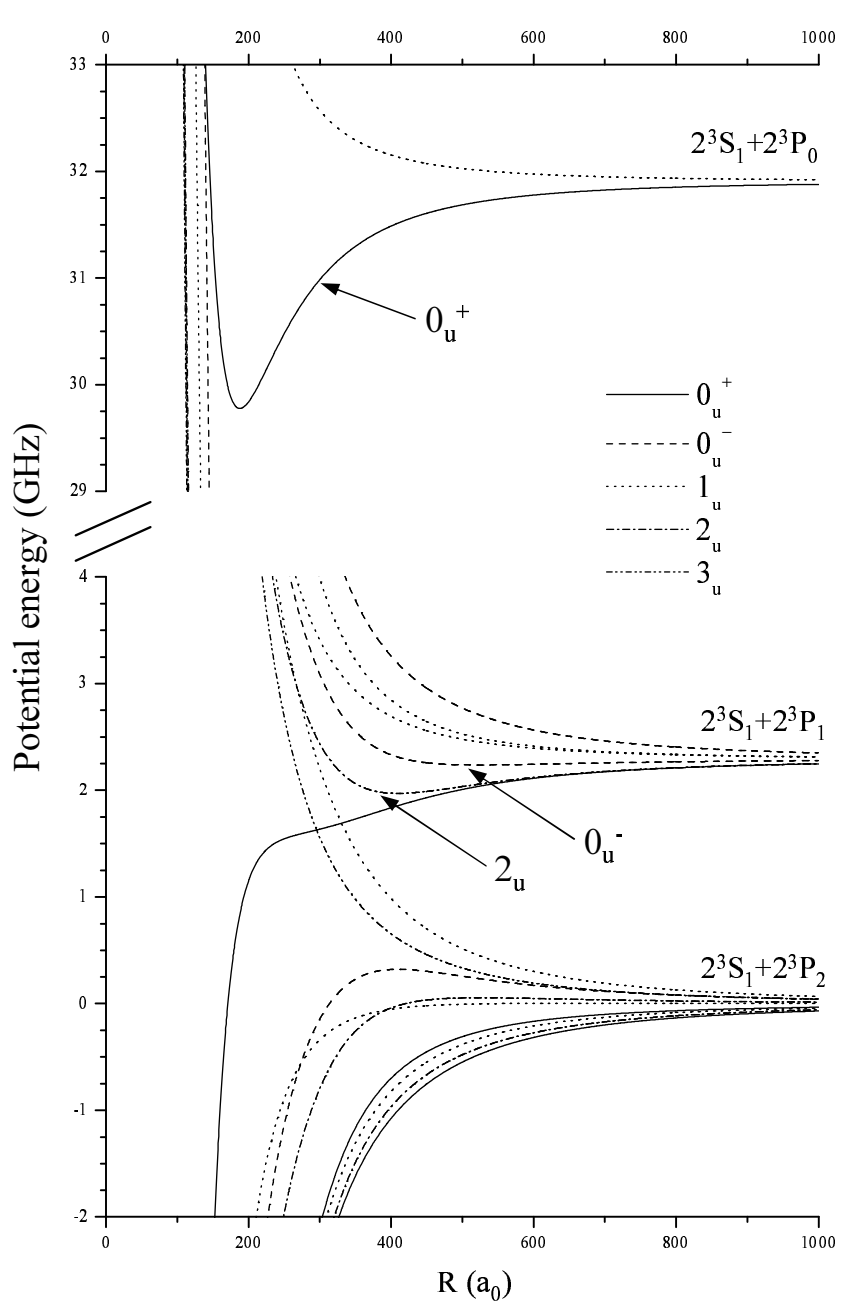

FIG. 5: Ungerade electronic potential curves (in $\mathrm{GHz}$ ) for fixed nuclei for the $2^{3} S+2^{3} P$ system versus the internuclear distance $R$ (in atomic units; $1 a_{0} \sim 0.0529 \mathrm{~nm}$ ). The potential curves result from the numerical diagonalization of the hamiltonian (13). Three arrows indicate the three purely long-range potential wells in which bound states are determined numerically.

phenomenologically, in order to reproduce the fine structure splittings which have been measured [20, 21] very accurately:

$$
\begin{gathered}
\alpha=-\frac{\Delta_{J=2 \leftrightarrow 1}}{2 \hbar^{2}} \quad \text { and } \quad \beta=\frac{2 \Delta_{J=1 \leftrightarrow 0}-\Delta_{J=2 \leftrightarrow 1}}{6 \hbar^{4}}, \\
\text { with }\left\{\begin{array}{l}
\Delta_{J=2 \leftrightarrow 1}=h \times 2.291175 \mathrm{GHz} \\
\Delta_{J=1 \leftrightarrow 0}=h \times 29.616950 \mathrm{GHz}
\end{array}\right.
\end{gathered}
$$

\section{Potential curves with fixed nuclei}

According to the Movre-Pichler approach [22], both retarded dipole-dipole interaction and atomic fine structure coupling:

$$
\hat{H}_{f s}(A)+\hat{H}_{f s}(B)+\hat{U}(R)
$$

should be considered simultaneously as a perturbation of the non relativistic hamiltonian for two independent atoms $\hat{H}_{0}(A)+\hat{H}_{0}(B)$. Only the projection $\Omega$ of the total electronic angular momentum on the molecular axis is a good quantum number. States of different $u / g$ symmetry are uncoupled and two sets of potential curves can be determined independently for gerade and ungerade states. Since we do photoassociation experiments in a magnetically trapped atomic cloud, the initial quasi-molecular state is ${ }^{5} \Sigma_{g}^{+}$, and gerade states are not accessible by single-photon excitation. Thus we focus only on ungerade states. Figure 5 shows the results of the calculated ungerade eigenvalues of the operator (13) as a function of $R$. Here, the electronic states are determined with fixed nuclei. Also, the potential curves describe only the longrange part of the molecular interactions as a consequence of the perturbative description. For the $\Omega=0$ space, the reflection symmetry (in a plane containing the molecular axis) leads to a relevant additional label $+/-$, which distinguishes two states with different energies. For $\Omega \neq 0$ states, this symmetry can be defined as well but the two resulting states have the same energy.

\section{Physical origin of the purely long-range molecules}

The hamiltonian (13) is block diagonal with each block corresponding to a given $\Omega_{u / g}^{( \pm)}$subspace. As an example, let us consider the subspace $0_{u}^{+}$. It is of dimension four. Figure [illustrates the physical reason why a purely longrange well arises in this subspace of states. If we consider only the dipole-dipole interaction, one eigenvalue is purely repulsive, while the three others are purely attractive, two of them being identical (Figure 6 a). They all have the same asymptote. The four corresponding eigenstates are pure Hund's case (a) states. Let us consider separately the repulsive state and the manifold of attractive states. If we "turn on" the fine structure coupling inside each of these two subspaces of states, while neglecting the couplings between them, then the potential curves repel each other and the asymptotes no longer coincide. Of course, since the neglected couplings are not small, the four asymptotes have no straightforward physical meaning. However, the important point is that a crossing shows up between the repulsive curve and one attractive curve (Figure 6 b). Finally, if we turn on the neglected fine structure terms, we couple the subspaces corresponding to the two crossing states, and an anticrossing appears (Figure 66. c). The resulting potential well is thus a consequence of the fine-structure mixing of long-range molecular interactions, which links the inner, repulsive dipole-dipole curve with an outer, attractive one. What is remarkable about this well is that even the repulsive part occurs at very long-range, in a region 
a)

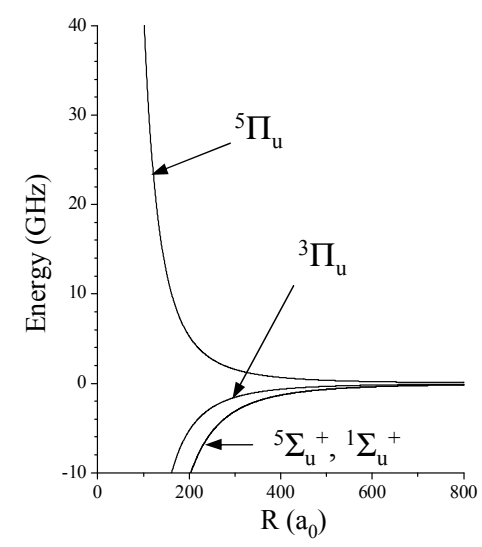

b)

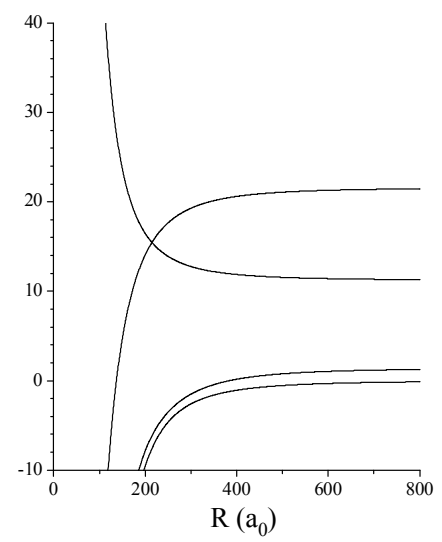

c)

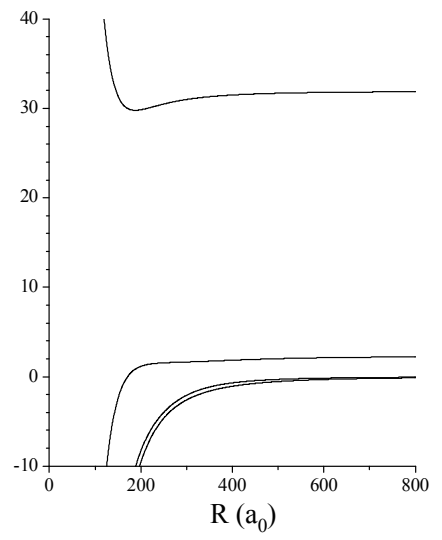

FIG. 6: Eigenvalues of the restriction of the hamiltonian 13 to the $0_{u}^{+}$subspace. Energies are given in GHz, distances are in atomic units. a) The fine structure coupling is neglected: the eigenstates are pure Hund's case(a) states. b) The fine structure coupling is partly included: couplings between the repulsive state and the attractive ones are neglected. After diagonalization, one attractive and one repulsive states cross each other. c) Finally, including all the fine structure coupling terms leads to an anti-crossing and a purely long-range potential well. Note that graph b) is only for illustration and that the neglected terms are not small.

where the asymptotic dipole-dipole expression remains a very good approximation. That is why the perturbative approach used here is very well suited to describe the bound states lying in this kind of well, or the so-called purely long-range molecular states [23].

Due to the competition between the dipole-dipole and the fine structure interactions, not only the potential curves, but also the electronic states explicitly depend on $R$. As an illustration, the $0_{u}^{+}$purely long-range electronic eigenstate is shown in Figure [7] The eigenstate is given with its projections over the Hund's case (a) basis set. It evolves from the pure Hund's case (a) ${ }^{5} \Pi_{u}$ at short

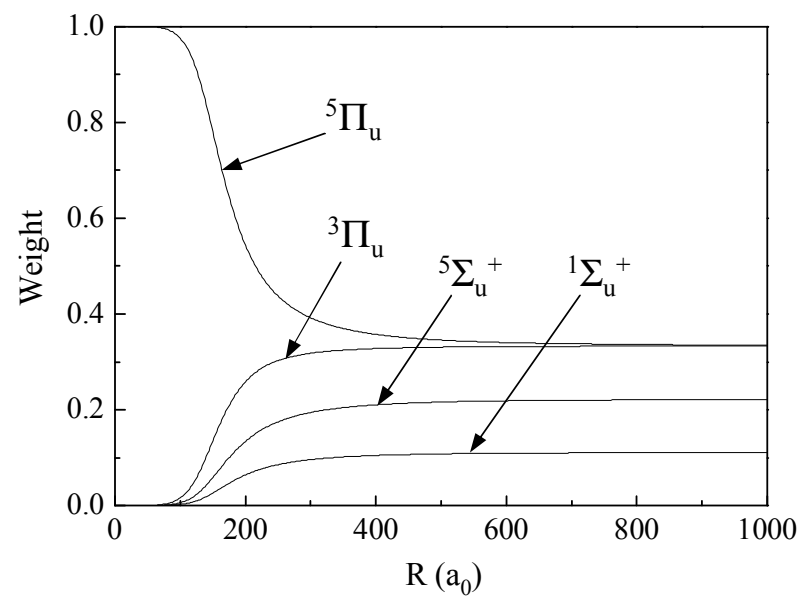

FIG. 7: Eigenstate for the $0_{u}^{+}$purely long-range potential well connected to the $2^{3} S+2^{3} P_{0}$ asymptote within the fixed-nuclei approximation. The electronic state is given with its decomposition in the Hund's case (a) basis set: the weights are the squares of the projection on the different subspaces of Hund's case (a) states. Distances are in atomic units. range, where the dipole-dipole interaction dominates, to a pure Hund's case (c) for asymptotically large values of $R$ where the dipole-dipole interaction vanishes like $1 / R^{3}$. Consequently, the fixed-nuclei approximation must be corrected by an accounting of the coupling between the electronic and nuclear degrees of freedom.

The discussion just presented can also be applied to all the other $\Omega_{u / g}^{( \pm)}$subspaces. Figure 5 shows three purely long-range ungerade potential wells. One is connected to the $2^{3} S_{1}+2^{3} P_{0}$ asymptote and belongs to the $0_{u}^{+}$ subspace; it has been presented above. The two others are connected to the $2^{3} S_{1}+2^{3} P_{1}$ asymptote and belong to the $0_{u}^{-}$and $2_{u}$ subspaces. Within the fixed nuclei approximation the calculated $0_{u}^{+}$well is $2.130 \mathrm{GHz}$ deep, the $2_{u}$ one is $0.321 \mathrm{GHz}$ deep, and the $0_{u}^{-}$one is 0.054 $\mathrm{GHz}$ deep. We will examine these wells more closely in the following discussion.

\section{B. Description of the motion of the nuclei}

So far the dynamics of the electrons has been treated independently from the dynamics of the nuclei. In our perturbative model, the coupling between the two comes from the kinetic energy operator for the relative motion of the nuclei:

$$
\hat{T}_{n}(R, \theta, \varphi)=-\frac{\hbar^{2}}{2 \mu}\left(\frac{1}{R} \frac{\partial^{2}}{\partial R^{2}} R-\frac{\vec{\ell}^{2}}{\hbar^{2} R^{2}}\right) .
$$

In this expression $(R, \theta, \varphi)$ are the spherical coordinates of the fictitious particle of reduced mass $\mu$ associated with the pair of nuclei, and $\vec{\ell}$ is the orbital angular momentum associated with its rotation. 



FIG. 8: Influence of the nuclear rotation on the electronic potential energy for the three ungerade purely long-range wells shown in Figure 5 The dotted lines are the result of the fixed nuclei approximation. The full lines are the potential used to calculate the binding energies presented in Table III Note that the horizontal and vertical scales are different for each graph.

\section{Effect of the rotation}

First, the effect of the rotation of the nuclei on the electronic states calculated above can be found if we add the last term of (14) to the hamiltonian (13). The operator to be diagonalized becomes:

$$
\hat{\mathcal{H}}=\hat{H}_{f s}(A)+\hat{H}_{f s}(B)+\hat{U}(R)+\frac{\vec{\ell}^{2}}{2 \mu R^{2}}
$$

Now, the space of states has to be extended to the rotational degrees of freedom. Only the total angular momentum $\vec{J}=\vec{L}+\vec{S}+\vec{\ell}$ has to be conserved [30], so we must consider the set of states $\left|\phi_{J, \Omega_{u}^{ \pm}}\right\rangle$defined by the product of electronic states determined above $\left|\Omega_{u}^{( \pm)}\right\rangle$and of rotational states $|J, M, \Omega\rangle[25]:\left|\phi_{J, \Omega_{u}^{ \pm}}\right\rangle=\left|\Omega_{u}^{( \pm)}\right\rangle \otimes|J, M, \Omega\rangle$. The quantum number $M$ is the projection of $\vec{J}$ onto a lab-fixed frame. Since the molecule is linear, $\vec{\ell}$ is orthogonal to the molecular axis, which means $\ell_{z}=0$ and $J_{z}=L_{z}+S_{z}$. Thus the electronic quantum number $\Omega$ represents the projection of $\vec{J}$ onto the molecular axis and it is recalled as a parameter in the notation for the rotational state. In this basis, $\vec{\ell}$ can be written as $\vec{\ell}=\vec{J}-\vec{L}-\vec{S}$, the square of which is given by:

$$
\begin{aligned}
\hat{\vec{\ell}}^{2}= & \hat{\mathbf{J}}^{2}+\hat{\mathbf{L}}^{2}+\hat{\mathbf{S}}^{2}-2 \hat{J}_{z}^{2}+2 \hat{L}_{z} \hat{S}_{z}+\left(\hat{L}_{+} \hat{S}_{-}+\hat{L}_{-} \hat{S}_{+}\right) \\
& -\left(\hat{J}_{+} \hat{L}_{-}+\hat{J}_{-} \hat{L}_{+}\right)-\left(\hat{J}_{+} \hat{S}_{-}+\hat{J}_{-} \hat{S}_{+}\right)
\end{aligned}
$$

In Equation (16), the first line contains terms that couple electronic states with each other inside each $\Omega_{u}^{( \pm)}$ block. The second line contains the terms that couple states belonging to different $\Omega$ subspaces, due to the action of $\hat{J}_{ \pm}$which obeys anomalous commutation rules 24] and couples $\Omega$ to $\Omega \mp 1$. These off-diagonal coupling terms become important where potential curves belonging to different $\Omega$ subspaces cross each other; they produce anti-crossings. For the three purely long-range wells of interest, such crossings appear far enough in the classically forbidden region that the off-diagonal coupling terms can be neglected in the calculation of the binding energy. Thus, in the following calculation, only the terms coupling states within a given $\Omega$ subspace (first line in Equation (16)) are kept in the expression of the rotation of the nuclei.

Figure 8 shows the change in the three ungerade potential wells resulting from the inclusion of the rotation of the nuclei in the hamiltonian. The minimum possible value for $J$ is $J=\Omega$. For higher values of $J$ the contribution of the centrifugal barrier due to the rotation of the nuclei increases. Bose-Einstein statistics dictates that $J$ should be odd for $0_{u}^{+}$, and even for $0_{u}^{-}$(see e.g. [25]). There is no restriction on $J$ for the $2_{u}$ state, since it is doubly degenerate.

\section{Effect of the vibration}

Next, since the electronic states depend on $R$ (Figure 77), the vibration of the nuclei also influences the electronic degrees of freedom. This effect is described by the radial part of the kinetic energy of the nuclei, namely the first term in parenthesis in Equation (14). This final addition to the hamiltonian leads to the following equation:

$$
\hat{H}|\psi\rangle=\left\{-\frac{\hbar^{2}}{2 \mu} \frac{1}{R} \frac{\partial^{2}}{\partial R^{2}} R+\hat{\mathcal{H}}\right\}|\psi\rangle=E|\psi\rangle
$$

where the eigenstates $|\psi\rangle$ are written using a basis with separable variables: $|\psi\rangle=\left|\chi_{v}\right\rangle \otimes\left|\phi_{J, \Omega_{u}^{ \pm}}\right\rangle$, with $\left|\chi_{v}\right\rangle$ the vibrational part, and $\left|\phi_{J, \Omega_{u}^{ \pm}}\right\rangle$the electronic and rotational part. With these notations, $\left|\phi_{J, \Omega_{u}^{ \pm}}\right\rangle$are the $R$-dependent eigenstates of the hamiltonian $\hat{\mathcal{H}}$, with the eigenvalues $V_{J, \Omega_{u}^{ \pm}}(R)$ determined previously and given in Figure 8

Because the crossings between electronic potential curves lie far enough in the classically forbidden region, 
the action of $\partial^{2} / \partial R^{2}$ on the electronic part should be considered as a diagonal correction and we neglect the off-diagonal terms of this operator. This is the so-called adiabatic approximation [14], and Equation (17) reduces to a set of independent radial equations:

$$
\left\{-\frac{\hbar^{2}}{2 \mu}\left(\frac{d^{2}}{d R^{2}}+\left\langle\phi_{J, \Omega_{u}^{ \pm}}\left|\frac{\partial^{2}}{\partial R^{2}}\right| \phi_{J, \Omega_{u}^{ \pm}}\right\rangle\right)+V_{J, \Omega_{u}^{ \pm}}(R)-E_{J, \Omega_{u}^{ \pm}, v}\right\} u(R)=0
$$

where the vibrational part of the wave function has been written $\left\langle\vec{R} \chi_{\chi_{v}}\right\rangle=u(R) / R$, and $E_{J, \Omega_{u}^{ \pm}, v}$ is the binding energy for the ro-vibrational level $(J, v)$ in the $\Omega_{u}^{ \pm}$potential well. Finally, the vibration of the nuclei is described through a single effective potential well which is the sum of $V_{J, \Omega_{u}^{ \pm}}(R)$ (which already takes into account the rotation of the nuclei) and of the correction coming form the dependence in $R$ of the eigenstates of $\hat{\mathcal{H}}$.

\section{Calculation and comparison with the experimental spectrum}

Table I provides the comparison between the experimental results obtained for the $0_{u}^{+}$potential well (column A), and the calculated binding energies from the adiabatic approach developed above (column B). In column (A), the measured binding energies include the corrections discussed in Section II Within the experimental accuracy, the agreement between our measurement and our predictions for $J=1$ is remarkably good (except for the $v=5$ line, which is too close to the atomic resonance to be observed). Note that the $v=0$ line was probed with a different laser set up, so its measured binding energy is less precise than the others (see [9]). Also, the $\left(0_{u}^{+}, J=3\right)$ progression is too weak to be observed in our experiment.

The effect of retardation on the calculated energy is illustrated by the quantity $\epsilon^{\text {Ret }}$ (Table \ column C). It increases the depth of the well, and therefore the binding energies as well. Compared with the non retarded calculation ( $k \rightarrow 0$ in the expressions 10a and 10b), retardation is a correction proportional to $R^{2}$ in relative value, but to $1 / R$ in absolute value. Therefore, it becomes very important in relative values for very elongated states (up to $\sim 30 \%$ for $v=5$ ), and it is more important in absolute values for less elongated states $\left(\epsilon^{\text {Ret }}=-6.6 \mathrm{MHz}\right.$ for $v=0)$. Given the experimental accuracy of $0.5 \mathrm{MHz}$, this work is a demonstration of the retardation effect, which has to be taken into account to reproduce the measured binding energies. This effect has been already demonstrated for sodium atoms in 1996 [27].

The correction to the electronic potential due to the vibration of the nuclei is illustrated by the quantity $\epsilon^{R a d}$ in Table【 column (D). Practically $\epsilon^{\text {Rad }}$ is the difference between the binding energy calculated with and without the
TABLE I: Comparison between experimental and theoretical binding energies in the case of the $0_{u}^{+}$purely long-range potential well. Column (A) gives the experimental results, after the corrections discussed in Section 1 are applied. Column (B) gives the binding energy $E_{v, J}$ calculated from equation 18 within the adiabatic approximation. For each bound state, $\epsilon^{\text {Ret }}$ is an estimate of the contribution to $E_{v, J}$ of the retardation effect. $\epsilon^{\text {Ret }}$ comes from the comparison with the non-retarded calculation. Similarly, $\epsilon^{\text {Rad }}$ is the calculated estimate of the term $\left\langle\phi_{J, \Omega_{u}^{ \pm}}\left|\partial^{2} / \partial R^{2}\right| \phi_{J, \Omega_{u}^{ \pm}}\right\rangle$(see Equation (18). Note that the binding energies presented in column (B) already implicitly contain the contributions $\epsilon^{R e t}$ and $\epsilon^{R a d}$. All the energies are given in units of $h$, in $\mathrm{MHz}$.

\begin{tabular}{cc|ccc}
\hline \hline & $(\mathrm{A})$ & $(\mathrm{B})$ & $(\mathrm{C})$ & $(\mathrm{D})$ \\
$v$ & Experiment & $E_{v, J}$ & $\epsilon^{\text {Ret }}$ & $\epsilon^{\text {Rad }}$ \\
\hline & & -2.487 & -0.78 & +0.053 \\
5 & - & -18.12 & -1.6 & +0.28 \\
4 & $-18.2 \pm 0.5$ & -79.41 & -2.6 & +0.95 \\
3 & $-79.6 \pm 0.5$ & -252.9 & -3.9 & +2.4 \\
2 & $-253.3 \pm 0.5$ & -648.3 & -5.2 & +5.3 \\
1 & $-648.5 \pm 0.5$ & -1418 & -6.6 & +10.3 \\
0 & $-1430 \pm 20$ & & & \\
\hline \hline
\end{tabular}

term $\left\langle\phi_{J, \Omega_{u}^{ \pm}}\left|\partial^{2} / \partial R^{2}\right| \phi_{J, \Omega_{u}^{ \pm}}\right\rangle$in Equation (18). This term is part of the kinetic energy of the system. Thus it brings a positive contribution to the effective electronic potential and it moves the bound states upward in the wells. Its contribution is non vanishing in the region where the electronic state changes its character with $R$ due to the anti-crossings discussed previously, that is to say in the vicinity of the bottom of the potential well. Therefore the correction is stronger for the deepest states, as they don't extend very far from this region. Weakly bound states extend much farther into regions where the electronic state does not depend strongly on $R$ (pure Hund's case $\mathrm{c}$ ), and the net effect is less pronounced.

Finally, the high accuracy of the data and the good agreement between the experimental and calculated spectra lead to an experimental determination of the $C_{3}$ coefficient, which describes the dipole-dipole interaction. In our calculations, changing $C_{3}$ by $0.1 \%$ changes the binding energies by at most $0.3 \mathrm{MHz}$, which is of order of our experimental accuracy. Therefore, the present results confirm the theoretical value used for the $C_{3}$ coefficient to within $0.2 \%$. As a consequence of Equation 11. we can infer that the atomic radiative decay rate is 
$\Gamma=2 \pi \times(1.625 \pm 0.003) \mathrm{MHz}$. As far as we know, this is the most accurate experimental determination for the helium $2^{3} P$ decay rate.

\section{Other ungerade giant dimers}

Bound states in ungerade potential wells other than $0_{u}^{+}$have not been explored. However, the calculation presented above can also be applied for those. Table II presents the theoretical results for the molecular binding energies and characteristic sizes in the three ungerade purely long-range potential wells. Column (A) gives the results obtained when one solves Equation 18 Experimentally, bound states are produced by driving an electric dipole transition from the electronic state ${ }^{5} \Sigma_{g}^{+}$ with $J=2$, so only $J=1,2$ or 3 are accessible. In Table III the results are given for one relevant value of $J$, taking into account the Bose-Einstein statistics already mentioned in paragraph आB 1

The purely long-range character of these molecules arises from the very large distance at which their inner classical turning points lie (Table II column B). The outer turning points (column $\mathrm{C}$ ) and mean sizes $\langle R\rangle=\left\langle\chi_{v}|R| \chi_{v}\right\rangle$ (column D) are also particularly large, leading to an unusual type of "giant" dimer for which asymptotic calculations allow an accurate description. At such large distances, the next order term $C_{6} / R^{6}$ in the electromagnetic interaction can clearly be neglected. The

TABLE II: Results of the calculation detailed in the text for the three purely long-range ungerade wells. Column (A) gives the binding energy $E_{v, J}$ calculated within the adiabatic approximation. The three last columns illustrate the unusual size of the dimers. $R_{\min }$ and $R_{\max }$ are classical inner and outer turning points, $\langle R\rangle$ is the mean internuclear distance. All the energies are given in $\mathrm{MHz}$, and the lengths in atomic units.

\begin{tabular}{|c|c|c|c|c|c|}
\hline & $v$ & $\begin{array}{c}(\mathrm{A}) \\
E_{v, J}{ }^{a} \\
\end{array}$ & $\begin{array}{r}(\mathrm{B}) \\
R_{\min } \\
\end{array}$ & $\begin{array}{r}(\mathrm{C}) \\
R_{\max } \\
\end{array}$ & $\begin{array}{l}\text { (D) } \\
\langle R\rangle\end{array}$ \\
\hline $0_{u}^{+}, J=1$ & $\begin{array}{l}5 \\
4 \\
3 \\
2 \\
1 \\
0\end{array}$ & $\begin{array}{l}-2.487 \\
-18.12 \\
-79.41 \\
-252.9 \\
-648.3 \\
-1418\end{array}$ & $\begin{array}{l}147.6 \\
147.7 \\
148.1 \\
149.5 \\
152.9 \\
162.5\end{array}$ & $\begin{array}{r}2182 \\
1122 \\
689 \\
467 \\
336 \\
246\end{array}$ & $\begin{array}{r}1797 \\
917 \\
560 \\
379 \\
276 \\
213\end{array}$ \\
\hline $0_{u}^{-}, J=2$ & 0 & -7.304 & 461.7 & 970 & 824 \\
\hline $2_{u}, J=2$ & $\begin{array}{l}3 \\
2 \\
1 \\
0\end{array}$ & $\begin{array}{l}-4.584 \\
-21.41 \\
-72.32 \\
-191.5\end{array}$ & $\begin{array}{l}320.5 \\
322.5 \\
329.3 \\
351.1\end{array}$ & $\begin{array}{r}2097 \\
1231 \\
808 \\
558\end{array}$ & $\begin{array}{r}1712 \\
999 \\
659 \\
477\end{array}$ \\
\hline
\end{tabular}

${ }^{a}$ Binding energies are given with respect to the asymptote of the potential considered.
$C_{6}$ coefficient has never been published for this system, but one can estimate that it is smaller than the value of $C_{6}=3265$ a.u. for the $2^{3} S-2^{3} S$ interaction 26] and calculate the order of magnitude of the neglected term. For internuclear distances larger than $150 a_{0}$, which is the range of interest for these purely long-range molecules (see Table【), $C_{6} / R^{6}<C_{3} / R^{3} \times 1.510^{-4}$. So neglecting this term leads to an error smaller than the one due to the uncertainty on $C_{3}$.

While writing the present article we were informed that Venturi et al. 28] had submitted for publication the result of a multichannel calculation, which is also in very good agreement with our experimental results. Their method is more elaborate and allows for a direct solution of the full set of equations (17). However, the binding energies obtained by both methods are equal to within 0.5 $\mathrm{MHz}$ for all the bound states presented in Table II We have also performed a multi-channel resolution of Equation 17 with the use of a mapped Fourier grid method. Our results 29] are comparable to those of reference 28] to within $100 \mathrm{kHz}$. The main reason why the adiabatic approach is efficient and the multi-channel calculation required is that there is no crossing between the adiabatic potential wells of interest and the other potential curves. This allows for a single-channel calculation that leads to Equation 18 and is accurate enough to reproduce the experimental spectrum.

\section{SUMMARY AND CONCLUSION}

In a previous Letter [9], we reported an accurate measurement of the binding energies of purely long-range helium dimers in the $0_{u}^{+}$potential well connected to the $2^{3} S_{1}+2^{3} P_{0}$ asymptote. The present paper reports theoretical calculations which complement the experimental results in order to interpret the spectra measured.

The experiment consists in measuring the PA laser detunings for which a strong heating of the atomic cloud is observed. The heating is assumed to be a consequence of the resonant excitation of a bound state in the $0_{u}^{+}$ potential well. To infer the corresponding binding energy, the measured PA laser detunings must be corrected from a mean shift of the molecular lines due to the nonzero magnetic field $B_{0}$ at the center of the trap, and also to the non-zero temperature of the cold gas. Since the detunings are measured with high accuracy, a simple calculation shows that the temperature-induced shift must be considered, given the range of temperature explored $(2-30 \mu \mathrm{K})$. This calculation does not include the exact shape and width of the lines but only gives in a mean correction. The binding energies deduced after correction are independant of the density, and no magnetic dipole moment is detectable for the excited state. Apart from the symmetric and asymmetric broadening mechanisms discussed in Section III the lineshapes are actually also influenced by the dynamics of the heating mechanism. Indeed the temperature curves are an indirect measure- 
ment of the lineshape which relies on the efficiency of the thermalization of the cloud. An incomplete thermalization can lead to another source of broadening of the lines, but no additional shift. The calorimetric detection scheme and its implications on the lineshape will be discussed in a separate paper.

Here we have presented an approximate solution of the Schrödinger equation that is well suited for asymptotically large internuclear distances. The adiabatic approach allows for accurate calculations of the binding energies in the case of purely long-range potential wells. The calculation can easily be extended to other purely long-range potential wells which can in principle also be observed in our experimental conditions, namely $0_{u}^{-}$and $2{ }_{u}$.

Finally, the comparison between the experimental and theoretical determination of the binding energies in the $0_{u}^{+}$potential well is very good if retardation effects are taken into account. As a consequence, an accurate measurement of the radiative decay rate for the excited atomic state $2^{3} P$ can be inferred. The accuracy of the experimental data allows for a test of retardation effects as well as of tiny vibration-induced couplings between electronic and nuclear degrees of freedom.

Thus, the excellent agrement between our perturbative calculation and our experiment suggests a good understanding of the purely long range system. This work is a first step towards a better knowledge of pair interactions in ultra-cold metastable helium. Further developments will follow in order to measure the s-wave scattering length for two atoms interacting through the ${ }^{5} \Sigma_{g}^{+}$ electronic potential.

Acknowledgements : The authors thank the group of F. Masnou-Seeuws, at Laboratoire Aimé Cotton in Orsay, for fruitful discussions.
[1] see e.g. review articles W.C. Stwalley,H. Wang, J. Mol. Spec. 195, 194 (1999), J. Weiner, V. S. Bagnato, S. Zilio, P. S. Julienne, Rev. Mod. Phys. 71, 1 (1999), F. MasnouSeeuws, P. Pillet, Advances in Atomic, Molecular, and Optical Physics 47, 53 (2001), and references therein.

[2] E. R. I. Abraham, W. I. McAlexander, C.A. Sackett, R. G. Hulet, Phys. Rev. Lett. 74, 1315 (1995).

[3] J. R. Gardner, R. A. Cline, J. D. Miller, D. J. Heinzen, H. M. J. M. Boesten, B. J. Verhaar, Phys. Rev. Lett. 74, 3764 (1995).

[4] N. Herschbach, P. J. J. Tol, W. Vassen, W. Hogervorst, G. Woestenenk, J.W. Thomsen, P. van der Straten, A. Niehaus, Phys. Rev. Lett. 84, 1874, (2000).

[5] F. Pereira Dos Santos, J. Léonard, Junmin Wang, C. J. Barrelet, F. Perales, E. Rasel, C. S. Unnikrishnan, M. Leduc, C. Cohen-Tannoudji, Phys. Rev. Lett. 86, 3459 (2001).

[6] A. Robert, O. Sirjean, A. Browaeys, J. Poupard, S. Nowak, D. Boiron, C. I. Westbrook, A. Aspect, Sci. Mag. 292, 463 (2001).

[7] O. Sirjean, S. Seidelin, J. Viana Gomes, D. Boiron, C. I. Westbrook, A. Aspect, G.V. Shlyapnikov, Phys. Rev. Lett. 89, 220406 (2002).

[8] M. Leduc, J. Léonard, F. Pereira dos Santos, E. Jahier, S. Schwartz and C. Cohen-Tannoudji, Acta Phys. Pol. B33, p. 2213 (2002).

[9] J.Léonard, M.Walhout, A.P.Mosk, T.Mueller, M.Leduc, C.Cohen-Tannoudji, Phys. Rev. Lett. 91, 073203 (2003).

[10] J. Stärck, W. Meyer, Chem. Phys. Lett. 255, p.229 (1994).

[11] F. Pereira dos Santos, J. Léonard, Junmin Wang, C. J. Barrelet, F. Perales, E. Rasel, C. S. Unnikrishnan, M. Leduc, C. Cohen-Tannoudji, Eur. Phys. J. D, 19, 103 (2002).

[12] R. Napolitano, J. Weiner, C. J. Williams, P. S. Julienne, Phys. Rev. Lett. 73, 1352 (1994).

[13] Ho Trung Dung, Ludwig Knöll, and Dirk-Gunnar Welsch, Phys. Rev. A 66, 063810 (2002).

[14] H. Lefebvre-Brion, R. W. Field, "Perturbations in the spectra of diatomic molecules", Acamdemic Press (1986).
[15] G. Herzberg, "Spectra of Diatomic Molecules", $2^{\text {nd }}$ edition (1950), D. Van Nostrand Company INC.

[16] E.I. Dashevskaya, A.I. Voronin, E.E. Nikitin, Can. J. Phys. 47, 1237 (1969).

[17] W. J. Meath, J. Chem. Phys. 48, 227 (1968).

[18] G. W. F. Drake in Atomic, Molecular and Optical Physics Handbook, edited by G. W. F. Drake, AIP Press, Chap.11 (1996).

[19] H. A. Bethe, E. E. Salpeter, "Quantum mechanics of oneand two-eletcron atoms", Springer-Verlag (1957).

[20] M.C. George, L.D. Lombardi, E.A. Hessels, Phys. Rev. Lett. 87, 173002, (2001), and references therein.

[21] J. Castillega, D. Livingston, A. Sanders, D. Shiner, Phys. Rev. Lett. 84, 4321, (2000), and references therein.

[22] M. Movre and G. Pichler, J. Phys. B, 10, p. 2631 (1977).

[23] W.C. Stwalley, Y.-H. Uang, G. Pichler, Phys. Rev. Lett. 41, p. 1164 (1978).

[24] see for example R. N. Zare, "Angular Momentum", Wiley and Sons (1988).

[25] J. T. Hougen, "The calculation of rotational energy levels and rotational line intensities in diatomic molecules", Nat. Bur. of Standards, momograph 115, (1970).

[26] J. Stärck, W. Meyer, Chem. Phys. Lett. 255, p.229 (1994).

[27] K. M. Jones, P. S. Julienne, P. D. Lett, W. D. Phillips, E. Tiesinga and C. J. Williams, Europhys. Lett., 35, 85 (1996).

[28] V. Venturi, P. J. Leo, E. Tiesinga, C. J. Williams, I. B. Whittingham, Phys. Rev. A. 68, 022706 (2003).

[29] M. Leduc, M. Portier, J. Léonard, M. Walhout, F. Masnou-Seeuws, K. Willner, A. Mosk, "Laser Spectroscopy XVI, Proceedings of the XVI International Conference on Laser Spectroscopy", Palm Cove, Australia (14-18 Juillet, 2003), Eds. P. Hannaford, H.-A. Bachor, K.G. Baldwin and A.I. Sidorov (World Scientific, New Jersey, London, Singapore, Hong Kong).

[30] Here, $\vec{L}$ and $\vec{S}$ represent the molecular orbital and spin angular momenta. 